\title{
CONSTRUCCIÓN EMERGENTE DEL CONCEPTO: CUIDADO PROFESIONAL DE ENFERMERÍA
}

\author{
María de Lourdes García Hernández", Lucila Cárdenas Becerril², Beatriz Arana Gómez³, Araceli Monroy \\ Rojas $^{4}$, Yolanda Hernández Ortega ${ }^{5}$, Cristina Salvador Martínez ${ }^{6}$
}

${ }^{1}$ Doutora en Ciencias de la Salud. Profesora de Tiempo Completo de la Facultad de Enfermería y Obstetricia de la Universidad Autónoma del Estado de México (UAEM). México. E-mail: luygaba@yahoo.com.mx

${ }^{2}$ Doutora en Ciencias de la Educación. Profesora de Tiempo Completo de la Facultad de Enfermería y Obstetricia de la UAEM. Integrante del Sistema Nacional de Investigadores de México. México. E-mail: lucycabe62@yahoo.com

${ }^{3}$ Doutora en Enfermería. Profesora de Tiempo Completo de la Facultad de Enfermería y Obstetricia de la UAEM. México. E-mail: betya18@yahoo.com.mx

${ }^{4}$ Doutora en Ciencias de la Educación. Investigadora de la Facultad de Enfermería de la Universidad Autónoma Metropolitana Xochimilco. Integrante del Sistema Nacional de Investigadores de México. México. E-mail: aramonroy@yahoo.com.

${ }^{5}$ Maestra en Enfermería. Profesora de Tiempo Completo de la Facultad de Enfermería y Obstetricia de la UAEM. México. E-mail: yolisho20@yahoo.com.mx

${ }^{6}$ Maestra en Enfermería. Profesora de asignatura de programa de Posgrado en la Facultad de Enfermería y Obstetricia de la UAEM. México. E-mail: cristina_901@hotmail.com.mx

RESUMEN: El objetivo del presente trabajo fue la construcción del concepto cuidado profesional de enfermería. Las aportaciones se encaminan a la dimensión profesional del campo de la ciencia de la enfermería. Es un estudio cualitativo con enfoque Hermenéutico, en 20 enfermeras, mediante entrevistas semiestructuradas a profundidad. El análisis fue de contenido. El cuidado profesional lo conceptualizan como el cuidado de la vida de las personas que solicitan sus servicios. Con ello, el hacer de la enfermera viene a ser un acto profesional y no un acto de buenas intensiones, con la obligación de asumir conductas de cuidado, que conlleva principios y normas establecidas por la disciplina. Las enfermeras entienden que se requiere de conocimientos constantes. La conceptualización del cuidado profesional que se apoya en la práctica, ofrece un acercamiento a la construcción de la Ciencia de la Enfermería.

DESCRIPTORES: Cuidado. Formación de concepto. Enfermería.

\section{EMERGING CONCEPT CONSTRUCTION: PROFESSIONAL NURSING CARE}

\begin{abstract}
The aim of this study was to help construct a professional nursing care concept. The contributions are aimed at the professional dimension of the science of nursing. This is a qualitative study with a hermeneutic approach involving 20 nurses in semistructured interviews submitted to content analysis. Professional care is conceptualized as caring for the lives of people who seek their services. Thus, the job of the nurse becomes a professional act and not an act of good intentions with the obligation of assuming care conduct, involving principles and standards established by the discipline. Nurses understand that knowledge requires continuous updating. The conceptualization of professional care based on practice offers an approach to the construction of the Science of Nursing. DESCRIPTORES: Care. Concept formation. Nursing.
\end{abstract}

\section{CONSTRUÇÃO EMERGENTE DO CONCEITO: CUIDADO PROFISSIONAIS DE ENFERMAGEM}

RESUMO: O objetivo deste trabalho foi a construção do conceito de cuidado profissional de enfermagem. As contribuições se encaminham a dimensão profissional do campo da ciência da enfermagem. É um estudo qualitativo com abordagem hermenêutica. Participaram 20 enfermeiras. Os dados foram coletados por meio de entrevistas semiestruturadas a profundidade e foi realizada análise de conteúdo. As enfermeiras conceitualizam o cuidado profissional como o cuidado da vida das pessoas que procuram seus serviços. Com isto, o fazer da enfermeira se torna um ato profissional e não um ato de boas intenções, com a obrigação de assumir condutas de cuidado que envolvem princípios e regras estabelecidas pela disciplina. As enfermeiras entendem que são necessários conhecimentos permanentes. A conceptualização do cuidado profissional -que se apoia na prática, oferece uma aproximação para a construção da Ciência da Enfermagem.

DESCRITORES: Cuidado. Formação de conceito. Enfermagem. 


\section{INTRODUCCIÓN}

Históricamente existen diferentes acepciones en relación al cuidado, por ejemplo Florence Nigthingale no definió atención o cuidado en su libro Notes of Nursing en 1859, Madeleine Leininger continua preguntándose si Nightingale consideró como componente del cuidado: el confort, ayuda y la educación de la salud. El cuidado como concepto debe ser entendido, " [...] debido a que los conceptos sirven como base para análisis y la investigación continua de la enfermera [... $]^{\prime 1: 81}$ considerando que en la actualidad el concepto de cuidado se cita con frecuencia para apoyar las practicas de las enfermeras actuales. El uso de la palabra cuidar involucra un sentido emergente, ya que, el concepto de cuidado se ha convertido en objeto de estudio de la enfermera en los últimos años. En enfermería el término cuidado se ha empleado de manera creciente desde la década de los años sesentas hasta nuestros días. ¿Pero, qué significa esta palabra?, el cuidado es una sensación que denota una relación de interés, cuando la existencia del otro te importa; una relación de dedicación, llevándolo a sus extremos, sufrir por el otro.

Cuidar como un concepto está en proceso de ser inventado o construido y se transforma con el paso del tiempo. El cuidado profesional es asumir una respuesta deliberada que envuelve un poder espiritual de afectividad. ${ }^{2}$ Este puede estar vinculado al uso de la tecnología y al grado de necesidad del cuidado del paciente, o sea, cuidamos de manera diferente a una persona que se encuentra en una unidad de cuidados intensivos que a otra que se encuentra en un ambulatorio, pero ambas reciben cuidados en grados diferentes. ${ }^{3}$ El cuidado profesional es desarrollado por profesionales con conocimientos científicos en el área de la salud, dotados de habilidades técnicas que auxilian a individuos, familias y comunidades a mejorar o recuperar la salud. ${ }^{2}$ Estas definiciones respaldan el acto de cuidar, sin embargo, dentro de los estudios ya realizados, se percibe lagunas en cuanto a las investigaciones que involucran la comprensión de los conceptos utilizados en la práctica de enfermería, especialmente cuando se trata del cuidado. Desde 1997 se hizo un llamado sobre la necesidad de que las enfermeras pusieran atención en la comprensión y no solamente en la explicación de los conceptos que guían la práctica profesional. ${ }^{4}$ En esta afirmación, se identifica el pensamiento de Martín Heidegger, al decir que la limitación de los conceptos no permite que se tenga una comprensión de su sentido original.
Lo anterior nos lleva a indagar ¿Cómo comprende la enfermera el concepto de cuidado profesional?, con el fin de reconocer la importancia y el origen de una estructura conceptual, fundamentada en la comprensión e interpretación de la propia experiencia de las enfermeras, situando el cuidado profesional como el hacer de la enfermera. Aunado a la contribución dentro del marco conceptual de la Ciencia del Cuidado.

La enfermera es la profesional que tiene la función primordial de preocuparse y priorizar el bienestar del paciente. La forma de interactuar en la atención es construida a partir de un complejo proceso de sensibilidad y reciprocidad, que se refieren a la vida y formas de fortalecer la relación de las personas. ${ }^{5}$

De ahí que el objetivo del presente trabajo fue la construcción del concepto "cuidado profesional de enfermería", para fundamentar la responsabilidad que conlleva el hacer de la enfermera. Las aportaciones se encaminan a considerar la dimensión profesional del campo de la enfermería, haciendo resaltar que la enfermera hace uso de conocimientos y habilidades con el fin de otorgar un cuidado profesional y que para hacerlo es indispensable entender el concepto de cuidado.

\section{METODOLOGÍA}

Para construir el concepto de cuidado profesional se escogió el estudio cualitativo para comprender aquello que conocemos y que tiene significado solamente en un contexto dado. El método hermenéutico sirvió de marco conceptual. El muestreo inicial fue intencional, localizándose 11 informantes clave, se continúo con un muestreo en cadena, llegando a un total de 31 casos confirmados, aplicando criterios de inclusión quedaron 20 enfermeras expertas en la práctica, considerándose aquellas que aplicaban sus conocimientos en cualquier campo de la práctica y realizaban cuidados en el paciente, conocimiento en el tema, más de tres años de antigüedad y experiencia en su campo laboral, estudios mínimos de licenciatura, que aceptaron gravar su conversación, concluir la entrevistada, firmar la carta de consentimiento informado. "[...] El factor de saturación de la muestra fue por la calidad de los datos y naturaleza del tema" .6:281

Las participantes se escogieron con base en los años de experiencia, considerándose a la enfermera experta como aquella que ha trabajado con situaciones de cuidado durante dos o tres años, cuenta con un bagaje de experiencia, comprensión 
intuitiva de cada situación y tiene en cuenta un gran número de diagnósticos y soluciones. Se utilizó una guía de entrevista semiestructurada a profundidad. ${ }^{1}$ El tiempo promedio fue de hora y media. Las respuestas fueron grabadas y registradas en una libreta de campo. De acuerdo con los conceptos de la filosofía del cuidado, se elaboró como pregunta orientadora del estudio: ¿Cómo conceptualiza el cuidado profesional?. Los datos se organizaron empleando el análisis de contenido de manera manual en las siguientes etapas: 1) pre análisis; 2) exploración del material; 3) reagrupamiento de categorías semánticas; 4) categorías temáticas; y 5) interpretación. ${ }^{7}$ Para presentar los datos, se utilizaron los componentes que construyeron las categorías sustantivas, constituidas por: comprendiendo el hacer de la enfermera; cuidar de la vida y la muerte como dualidad; el cuidado como una enseñanza de vida; y fundamentar la ciencia del cuidado. Se recurrió a dos jueces en la temática para garantizar la credibilidad del estudio y asegurar los relatos de los entrevistados. Una revisó las categorías encontradas y la coherencia con los discursos de los entrevistados y otra analizó el contenido de las subcategorías. Esto reforzó la credibilidad del estudio. El aspecto ético de la investigación se fundamentó en el artículo 13 y 14, Fracc. V, de la Ley General de Salud Mexicana 2000. Fue evaluado por el Comité de Enseñanza, Investigación y Ética del Hospital Centro Médico Adolfo López Mateos, con el registro EN/01/1110.

\section{PRESENTACIÓN Y DISCUSIÓN DE RESULTADOS}

\section{Descripción de las participantes}

Los informantes clave se localizaron en cinco entidades Federativas: siete enfermeras en el Distrito Federal; ocho en el Estado de México; dos en Puebla y dos en Chihuahua; y una en Guanajuato. Ellas han obtenido conocimientos con Doctoras de Universidades de: Brasil, Colombia y Estados Unidos, en donde han realizado estudios sobre el cuidado. El nivel de estudios de las enfermeras fueron: ocho licenciadas, cinco con maestría, con doctorado cinco y dos con especialidad. Los años de experiencia se consideraron de acuerdo a la definición de enfermera experta que la tipifica como: aquella que requiere trabajar con situaciones de cuidado durante dos o tres años. ${ }^{1}$ Con base a ello, cinco de las enfermeras tenían entre 11 a 15 años de experiencia; otras cinco de 26 a 30 años; tres entre seis y 10 años; dos con 16 a 20; dos de 21 a
25; dos entre 31 a 35, y sólo una contaba con tres años de experiencia. En promedio las participantes contaban con 16 años de experiencia.

Las instituciones en donde laboraban las informantes fueron: siete de ellas en Facultades de Enfermería de universidades públicas; cuatro en Hospitales y Centros de salud del Instituto de Salud del Estado de México (ISEM); tres en el Instituto de Seguridad y Servicios Sociales de los Trabajadores del Estado (ISSSTE); y dos informantes clave en cada una de las siguientes instituciones: Instituto Mexicano del Seguro Social (IMSS); Hospitales de tercer nivel de la Secretaría de Salud (SS), Hospitales de segundo nivel integrados en el Desarrollo Integral de la Familia (DIF); Hospitales del Instituto de Seguridad Social del Estado de México y Municipios (ISSEMYM), Industrias; y una en un hospital privado. Participaron más enfermeras de instituciones educativas, sin embargo, cinco de ellas laboraban al mismo tiempo en hospitales y dos trabajaron en hospitales por más de 15 años. El sector en que laboraban fueron instituciones públicas y privadas de diferentes niveles de atención: once en un segundo nivel de atención, seis en tercer nivel y tres en primer nivel. Las enfermeras denotan haber obtenido habilidad y experiencia en su mayoría en hospitales de segundo y tercer nivel. Las áreas de habilidad y expertez: siete de las enfermeras practican la docencia en asignaturas de epistemología, cuidado y proceso de enfermería, seis en el servicio de medicina interna, gineco obstetricia, quirófano y cirugía, en estas, dos enfermeras por cada uno de los servicios; una en terapia intensiva y neurología, el resto en áreas de prevención. Las características de las informantes claves permiten visualizar una formación profesional en el conocimiento y experiencia del tema.

\section{Comprendiendo el hacer de la enfermera}

En la actualidad, las enfermeras definen el cuidado; en acciones dirigidas a lograr la recuperación de la salud del paciente, sin olvidar el origen o residencia y la cultura en el que se desarrolla. Las enfermeras comprenden que los cuidados integrales los da una enfermera profesional que practica la enfermería y que tiene la responsabilidad del cuidado del paciente, entendiéndose como profesional, a una enfermera con habilidades, actitudes, valores culturales y éticos, necesarios, para practicar la enfermería.

[...] yo entiendo el cuidado profesional como las acciones encaminadas al paciente para lograr su bienestar, identificando necesidades, valorando lo que se le 
tiene que hacer, sin olvidar su cultura o etnia a la que pertenece $[. .].(\mathrm{E}-18)$.

La enfermera se remite a un cuidado que se basa en la cultura de la persona que recibe el acto de cuidar, relacionando esta forma de pensar con el concepto de Leininger sobre la teoría de la diversidad y universalidad del cuidado. De ahí que el cuidado siempre será único y diverso en cada paciente; ya que se desarrollan en diversas etapas de vida y de culturas distintas, es decir:

[...] cuidar profesionalmente es una acción que podemos realizar en toda la diversidad de estados del ser humano, en un estado de enfermedad física, mental, rehabilitación, nunca dejamos de cuidar la salud [...] (E-14).

[...] definitivamente el cuidado profesional es nuestro monopolio, la enfermera es la única que sabe cuidar, de manera integral [...] (E-11).

El cuidado va intrínsecamente relacionado con la "[...] curación, la ética y la escala de valores de cuidado, curación, y salud; comprenden el contexto profesional, la misión de la enfermera y su razón de ser para la sociedad" ${ }^{9: 51}$ La conceptualización de cuidado es entendida como la misión de la enfermera y su razón de ser para la sociedad, se observa en lo que refieren las enfermeras, sobre la definición del cuidado como una acción monopólica, y que, la enfermera es la profesional que da diversos cuidados integrales al individuo en cualquier nivel de salud en que se encuentre. ${ }^{9}$

\section{Cuidar de la vida y la muerte como una dualidad}

Las prácticas primitivas del cuidado de la vida, de la preservación de la salud y de la curación de las enfermedades se han venido transformando con el paso del tiempo, a partir de la consideración de los nuevos descubrimientos en ciencia y tecnología, de los cambios sociales, políticos, culturales y económicos; de la globalización e internacionalización en la oferta de bienes y servicios y de la llamada revolución científica o era del conocimiento, sin embargo, los actos humanos siguen presentes. Por ejemplo, el cuidado de la vida inicia desde la etapa intrauterina, continúa durante todo el proceso biológico del ser humano y concluye con la muerte. Leonardo Boff, fundador de la Teología de la Liberación, da a conocer que el cuidado es esencial, y que es un acompañamiento permanente en el ser humano. Bajo este punto de vista surge el cuidado con un enfoque social, en donde inician las ligazones entre lo espiritual, material, social, humano y cultural. ${ }^{10}$ Estas conexiones se dan mediante un cuidado o descuido, de donde surge la vida, y que en ese continuo, se transforma en enfermedad.

Cuando el hombre tiene dolor en alguna parte de su cuerpo, no sólo es éste, sino es todo el ser humano que sufre ese dolor, angustia e incertidumbre. En su dimensión social, se aísla, deja de trabajar, y se tiene que cuidar. La curación es un proceso global, que implica la totalidad del ser humano y no sólo la parte enferma. ${ }^{11}$ Esclepio, héroe sanador de Grecia, tuvo tanta eficacia en sus métodos, que tras su muerte fue divinizado. Afirmaba que la curación sólo es completa cuando resulta de la intervención humana. El cuidado de la vida en la historia, se encuentra ligado a la mujer, quien se encargaba de mantener la salud de la familia. Por tradición, las comadronas o llamadas también parteras, se relacionan con el cuidado de la vida de la parturienta y el niño. Esto confirma que los seres humanos siempre tienen necesidad de cuidarse y ser cuidados, por ello, cuidar, se considera un acto de vida. La orientación de los cuidados basados en todo lo que contribuye a asegurar el mantenimiento y la continuidad de la vida nace de la Physis, es decir, la ciencia de la naturaleza. El cuidado forma parte de la naturaleza humana. ${ }^{12}$

Cuando aparece la vida sedentaria, la enfermedad era considerada un acto divino, un castigo de los Dioses; por ello, se crea lo bueno y lo malo y se confía al chaman el cuidado de la vida y luego al sacerdote. Con la llegada de la tecnología, a finales del siglo XIX, el cuidado se orientó más al restablecimiento de la complejidad biológica. Esto generó que el cuidado se aislara y abandonara, se cortó - parcializó-, se generó el cuidado incompleto, hasta convertirse en cuidar la enfermedad. Se pierde el cuidado de la vida. ${ }^{13}$ La mujer, como madre, deja de ser útil en el cuidado de la vida, porque se crea el cuidado de la enfermedad. Se requieren personas que se preparen para lograr que el ser humano recupere la salud, de ahí, surge la enfermera. Ésta da dos orientaciones al cuidado; uno de naturaleza hacia la vida y otro hacia los heridos y enfermos, ${ }^{14}$ así lo refieren las entrevistadas:

[...] el cuidado profesional es proporcionar una atención de cuidado integral al individuo; es prevenir que el paciente tenga una enfermedad [...] (E-5).

Cuidar el cuerpo humano enfermo o discapacitado ha sido por mucho tiempo el campo de acción de enfermería. En la actualidad las enfermeras identifican el cuidado como atender y prevenir, dejando de lado la ocasión real de cuidado y el momento del cuidado transpersonal, fenómenos 
que ocurren cuando existe una relación de cuidado auténtico entre la enfermera y el paciente, con el fin de realizar acciones encaminadas a la prevención, en la enfermedad o la rehabilitación del paciente, considerando que el cuidado lo entienden como:

[...] cuidado profesional es proporcionar los cuidados integrales al paciente desde el inicio de su padecimiento, hasta su recuperación, también se dan cuidados, aunque no se esté enfermo, ya que, se tiene que trabajar profesionalmente para mantener a la población sana [...] (E-5).

En este caso la enfermera dimensiona que el cuidado se debe de direccionar hacia la salud y no a la enfermedad. Este pensamiento se respalda en la concepción sobre las acciones efectivas al paciente/ familia, que requieren de un apoyo mutuo y atento en una comunidad moral de profesionales que buscan crear y mantener una buena práctica proporcionando cuidados integrales. ${ }^{1}$ La concepción del cuidado que considera el sujeto en sus múltiples dimensiones, y que utiliza los sentidos para buscar informaciones importantes, debe ser valorizada, sin embargo, es de extrema importancia la aplicación del conocimiento técnico-científico bien fundamentado y la presencia de un profesional bien preparado, sobre todo en los escenarios de tecnología de punta, promoviendo un equilibrio entre el cuidado expresivo y el técnico/tecnológico. ${ }^{15}$

\section{El cuidado como una enseñanza de vida}

Las enfermeras han alcanzado áreas del conocimiento útiles para llevar a cabo el cuidado, siguiendo patrones que han sido establecidos en la salud pública, la epidemiología, la fisiología, la biología y la psicología por mencionar algunas disciplinas, estas fundamentan parte del conocimiento y enseñanza del cuidado a los pacientes, es decir:

[...] cuidar profesionalmente es enseñar recomendaciones de salud para que el ser humano tenga una calidad de vida [...](E-4).

Las enfermeras expresan que enseñar es cuidar y lo relacionan con la vida de las personas. Enseñar es una relación entre el paciente y la enfermera que se da mediante la comunicación y la interrelación comprometida, como lo mencionó Nightingale en sus escritos, en donde pide a sus lectores ponerse imaginariamente en el sitio del inválido para considerar los efectos del comportamiento de otros y de ellos mismos sobre él. Ella solicita algo que ahora llamamos imaginariamente empatía, la capacidad de ponerse a uno mismo imaginariamente y sensitivamente en el mundo del otro. ${ }^{16}$ " [...] El cuidado como una forma educativa de relacionarse con un ser apreciado hacia el que siente un compromiso y una responsabilidad personal" ${ }^{17: 163} \mathrm{El}$ cuidado es una relación directa con el paciente, mediante la educación, ${ }^{16}$ éste se ve reflejado en el pensamiento que expresan las participantes al decir que cuidar es enseñar y que se tiene que llevar a cabo el cuidado tanto la en población sana y enferma, por lo tanto, el cuidado es enseñanza, en donde se utilizan los conocimientos relacionados con la profesión.

Realmente el cuidado profesional de enfermería al paciente, es poder ofrecer los conocimientos que tengamos para que él pueda recuperar su salud, enfermería es cuidado [...] (E-5).

Algunas veces se habla del cuidado como si para realizarlo no se necesitara de conocimiento, como si cuidar a alguien, fuera algo tan simple. Para cuidar a alguien, debo de saber, quién es a la persona que se cuidará, cuáles son sus necesidades, que me conduce a querer cuidarlo. Cuidar requiere de un estudio serio, reflexivo, de acción y una búsqueda de conocimientos y nuevas ideas que ayuden en el proceso de cuidado durante sus experiencias de salud y enfermedad. ${ }^{18}$ Ofrecer los conocimientos al paciente es nutrir a las personas que viven el cuidado y que crecen en el cuidado. ${ }^{19}$ Los cuidados son el objetivo de la ciencia de la enfermería y que la enfermera debe de estar constantemente alimentando al ser humano de cuidados, en este sentido las enfermeras ofrecen cuidados utilizando conocimientos, considerándolo como:

[...] un estado de equilibrio de armonía, [...] contrario a la enfermedad, que es un estado de desarmonía $y$ desequilibrio de la persona que enferma [...] (E-14).

El desequilibrio lo podemos entender como un proceso reparador, no necesariamente acompañado de sufrimiento. Cuidar son acciones que realizan las enfermeras con la intensión de que los individuos desarrollen al máximo sus capacidades para mantener, conservar la vida y permitir que ésta continúe considerando sus derechos y sentimientos. ${ }^{20}$ En el 2007, se definió el cuidado como la respuesta humana de la persona, familia y/o comunidad tanto sana como enferma, en los aspectos biológico, psicológico y social. ${ }^{21}$

\section{Fundamentar la ciencia del cuidado}

Con lo anterior se puede mencionar que: [...] realmente el cuidado profesional es el punto de partida de la ciencia del cuidado, lo que significa, que la enfermera debe de saber por qué lo hace, para qué lo hace, cómo lo 
hace y cuándo lo hace, esa es la diferencia de un cuidado profesional de enfermería de uno que no loes [...] (E-11).

Por lo tanto, el cuidado se enfoca específicamente a que las enfermeras profesionales sean las responsables de planificar y ejecutar cuidados. Ellas deben de saber, que cuidados deben de ofrecer desde que están en contacto con el sujeto que solicita el cuidado. Así hablar de un método para su aplicación, nos lleva a la reflexión de que se requiere de un marco conceptual para construir la organización de los mismos.

Cuidar es, ante todo, un acto de vida, en el sentido de que representa una infinita variedad de actividades dirigidas a mantener y conservar la vida y permitir que ésta continúe y se reproduzca. Es un acto de reciprocidad que se tiende a dar a cualquier persona que, temporal o definitivamente, requiere ayuda para asumir sus necesidades vitales.

[...] cuidar profesionalmente para mí es el objeto de la profesión, pero no está siendo comprendido, existe una falta de comprensión que produce problemas en la significación del cuidado y eso tiene trascendencia dentro de nuestra profesión, porque no se conoce bien, el concepto de lo que es cuidar. Pues, se cuida mucho la parte de la enfermedad [...] (E-11). Aunque el [...] término cuidado, es sumamente amplio, para mí cuidado es la esencia de la enfermería [...] (E-7).

Analizando estas respuestas se puede entender que el concepto es parte del lenguaje de la enfermera, aunque aún no es comprendido, se identifica que para ellas, es un término entendido en cada acción que realizan. Las enfermeras reconocen y dan significado al término de cuidado como la esencia de la profesión, sin embargo, la incomprensión sobre lo que es el cuidado trasciende en los cuidados que la enfermera proporciona al paciente en la actualidad.

Con base en lo anterior, para proporcionar un Cuidado Profesional se requiere de: conocimientos, actitudes y destrezas que sólo se adquieren con el estudio profundo y sistemático de la sociedad, del hombre, la salud y la propia disciplina de enfermería. Para que los cuidados se presten con profesionalismo, las enfermeras deben: entender al hombre de forma integral, tener bien clarificadas las acciones y metas, utilizar una metodología de trabajo, basar sus acciones en principios científicos, poseer capacidad para responder a una obligación ética y legal que han contraído.

El sujeto del cuidado, indiscutiblemente, es el ser humano. Las enfermeras, como profesionales de la disciplina, igualmente prestan sus servicios a grupos humanos (familia y comunidad). El cuida- do de la salud, desde la perspectiva enfermera, se enfoca partiendo de una visión global del hombre, considerando los aspectos fisiológicos, psicológicos y sociales que están presentes en todas y cada una de las personas. ${ }^{22}$

Bajo este panorama el concepto del cuidado ha transitado en tres etapas: El cuidado de la vida, cuidado del paciente como humano y el cuidado aprendido por la práctica, fundamentado el conocimiento epistémico de la enfermera. Con ello se establece que el Cuidado Profesional de Enfermería se define, como: la esencia de la disciplina, tiene como fin el cuidado integral del paciente en condiciones de salud y enfermedad, mediante acciones de cuidado y enseñanza en el mantenimiento y conservación de la vida, con conocimientos científicos y humanísticos que permiten mantener el bienestar del ser

\section{CONSIDERACIONES FINALES}

Las definiciones expuestas por las enfermeras sobre el concepto de Cuidado Profesional de Enfermería, nos colocan en el punto central de la reflexión, sobre lo que hace una enfermera. Conceptualizan el cuidado profesional como un cuidado de la vida de las personas que solicitan sus servicios. Con ello, el hacer de la enfermera viene a ser un acto profesional y no un acto de buenas intensiones, ya que tienen la obligación moral y ética de asumir conductas de cuidado, con principios y normas establecidas por la disciplina de enfermería. Las enfermeras entienden que un cuidado profesional es aplicar sus conocimientos en cada una de sus acciones y que estas conllevan la enseñanza y el aprendizaje continuo del cuidado.

Partiendo de lo anterior podemos conceptualizar que el cuidado profesional es entendido como la esencia de la disciplina de enfermería y tienen como fin cuidar integralmente al ser humano en condiciones de salud y enfermedad mediante enseñanzas de cuidado, sustentadas en un conocimiento teórico-práctico científicos y humanísticos para conservación y mantenimiento de la vida.

Finalmente, la construcción del concepto de cuidado profesional que se apoya en la práctica ofrece un acercamiento a la construcción del marco de referencia de la Ciencia de la Enfermería.

\section{REFERENCIAS}

1. Marriner A, Raile MA. Modelos y teorías en enfermería. España: Elservier Mosby; 2007. 
2. Waldof VR. Cuidado humano. $2^{\mathrm{a}}$ ed. Porto Alegre (RS): Sagra Luzzato; 1999.

3. Rocha PK, Prado ML, Gasperi P, Sebold LF, Waterkemper R, Bub MBC. El cuidado y la enfermería. Av Enferm Colombia. 2009 Sep; 27(1):102-9.

4. Silva FL, Coelho DMM, Lima CCM, Silva SPD. Cuidados de enfermería: su sentido para enfermeras y pacientes. Rev Enferm de Chile. 2002; (12):1-9.

5. Bazotti KDV, Stumm EMF, Kirchner RM. Ser cuidada por profissionais da saúde: percepções e sentimentos de mulheres que sofreram abortamento. Texto Contexto Enferm. 2009 Jan-Mar; 18(1):147-54.

6. Burns N, Grove SK. Investigación en enfermería. $3^{\text {a }}$ ed. España: Elservier; 2004. p.281-91.

7. Rodrigues MSP, Maria TL. O método de análise de conteúdo: uma versão para enfermeiros. Fortaleza (CE): Fundação Cearense de Pesquisa e Cultura; 1999. p. 99-112.

8. Fernández JA, Barajas G, Barroso L. Profesión, ocupación y trabajo: Eliot Freidson y la conformación del campo. México: POMARES; 2007.

9. Watson J. The theory of human caring: retrospective. Nurs Sci Q. 1997 Marc; 10(1):49-52

10. Maturana H. La realidad: ¿ objetiva o construida?. Fundamentos biológicos de la realidad. Universidad Iberoamericana. México: Anthropos Editorial; 1997.

11. Boff L. El cuidado esencial. Madrid (ES): Tratta; 2002.

12. Morín E. Introducción al pensamiento complejo. Barcelona: Gedisa; 2001.
13. Aimar R, Videla N, Torre M. Tendencias y perspectivas de la ciencia enfermería. Enfer Global [internet]. 2006 Nov [citado 2010 Sep 19]; (9): Disponible: http:// revistas.um.es/eglobal/article/view/385

14. Sabatier P. Knowledge, policy-oriented learning, and policy, change. Sci Communication. 1987 Jun; 8(4):649-62.

15. Silva RC, Ferreira MA. Una nueva perspectiva sobre el conocimiento especializado en enfermería: un debate epistemológico. Rev Latino-am Enfermagem [online]. 2008. Nov-Dez [cited 2008 Nov 16]; 16(6): Disponíble en: http://www.scielo.br/pdf/rlae/ v16n6/pt_17.pdf

16. Dunlof MJ. Interpretative phenomenology. California. United States of America: SAGE; 1994.

17. Swanson KM. Empirical develoment of a middle range theory of caring. Nurs Res. 1991 May-Jun; 40(3):161-6.

18. Watson J. Enfermería: ciencia humana y cuidado humano, una teoría en enfermería. Colorado United States of America. 1988.

19. BoykinS. Nursing as caring: a model for transforming practice. New York (US): National League for Nursing Press; 1993.

20. Colliere MF. Promover la vida. Madrid (ES): Interamericana; 1993.

21. Casanova E. Ciclo del crecimiento de los conocimientos de enfermería. Desarrollo Científ Enferm. 2006 Nov-Dic; 11(10):454-60.

22. García R. El conocimiento en construcción. Barcelona (ES): Gedisa; 1995. 